\title{
CONOCIMIENTOS, ACTITUDES Y PRÁCTICAS DE LACTANCIA MATERNA DE MADRES QUE ACUDEN AL CONTROL PRE-NATAL EN UN HOSPITAL DE ICA-PERU.
}

\section{KNOWLEDGE, ATTITUDES AND PRACTICES OF BREASTFEEDING OF MOTHERS WHO COME TO PRE-NATAL CONTROL IN A HOSPITAL OF ICA-PERU.}

\section{ARTÍCULO ORIGINAL} \author{
Cosme Macera Barriga ${ }^{2, b}$ \\ 1. Médico Pediatra \\ 2. Medicina Humana \\ a. Hospital Santa María del Socorro \\ b. Facultad de Medicina Humana, Universidad Nacional San Luis Gonzaga de Ica
}

Manuel Antonio Injante Injante ${ }^{1, a, b}$, Guadalupe Aracelia Alvarez Diaz ${ }^{2, b}$, Lisbeth Melissa Gavilano Bernaola ${ }^{2, b}$,

\section{Correspondencia:}

Cosme Macera Barriga.

Dirección: Urb. Torres san Joaquin

Bloque I-101- Ica, Perú.

Teléfono: (51) 987848894

Correo Electrónico:

cosme.macera@Gmail.com

Contribuciones De Autoría: MAll, GAAD, LMGB, CMB participaron en el diseño del estudio, el análisis de los datos, revisaron críticamente el artículo y aprobaron la versión final

Conflicto De Intereses: No declarados.

Financiamiento: Autofinanciado.

\section{Como Citar}

Injante Injante $\mathrm{M}$, Alvarez Díaz $\mathrm{G}$, Gavilano Bernaola L, Macera Barriga C. Conocimientos, actitudes y prácticas de lactancia materna de madres que acuden al control pre-natal en un hospital de Ica-Perú. . Rev méd panacea. 2017;6 (2): $53-59$

Recibido: 10-05-2017

Aceptado: 15-06-2017

Publicado: 20-06-2017

\begin{abstract}
RESUMEN:
Introducción: La leche materna constituye el alimento natural e ideal, el más completo, que le ofrece la naturaleza al individuo, desde el mismo instante de su nacimiento; aportando múltiples beneficios; tanto al recién nacido como a la madre. Por otra parte, las repercusiones de la falta de leche materna duran toda la vida, incluyendo dificultades de desarrollo intelectual, cognitivo y social; siendo la prevalencia mundial de lactancia materna baja, aumentando significativamente el riesgo de morbilidad y mortalidad cuando un infante menor de seis meses de edad no es alimentado exclusivamente con leche materna. Objetivo: Evaluar los conocimientos, identificar las actitudes, determinar las prácticas y revisar la congruencia entre estos tres puntos referentes a lactancia materna exclusiva. Metodos de Estudio: Nivel de Investigación observacional, tipo analítico, prospectivo y transversal. Realizado mediante frecuencias y un análisis de asociación mediante chi2 - 95\% Resultados: Se concluye que las gestantes tienen buenos conocimientos de lactancia materna exclusiva, sin embargo estos conocimientos no son coherentes con las actitudes y prácticas identificadas.
\end{abstract}

Palabras Claves: Lactancia Materna, Lactante, Atención prenatal.

\section{ABSTRACT:}

Introduction: Breast milk constitutes the natural and ideal food, the most complete, offered by nature to the individual, from the moment of birth; where they contribute benefits; As well as to the mother. On the other hand, the repercussions of the lack of breast milk last a lifetime, the difficulties of intellectual, cognitive and social development, being the global prevalence of breastfeeding low, increasing the risk of morbidity and mortality when an Infant less than six months old is not exclusively breastfed. Objetive: Evaluate knowledge, identify attitudes, identify practices, determine the practice and to check the congruence between these three points referring to exclusive breastfeeding. Methods of Study: Level of observational research, analytical, prospective and cross-sectional type. Performed by frequency and association analysis using chi2 95\% Results: Concluding that pregnant women have knowledge about raw materials and practices.

Keywords: Breast Feeding, Infant, Practice. 


\section{INTRODUCCIÓN}

La leche materna constituye el alimento natural e ideal, el más completo, que le ofrece la naturaleza al individuo, desde el mismo instante de su nacimiento; aportando múltiples beneficios; tanto al bebé como a la madre (1).

Por otra parte, las repercusiones de la falta de leche materna duran toda la vida, dificultades de desarrollo intelectual, cognitivo y social, siendo la prevalencia mundial de lactancia materna baja, aumentando significativamente el riesgo de morbilidad y mortalidad cuando un infante menor de seis meses de edad no es alimentado exclusivamente con leche materna(1). Ante este escenario, la Organización Mundial de la Salud (OMS) y la UNICEF desde el año 1991 lanzaron la Iniciativa Hospital Amigo del Nino (IHAN) buscando fomentar los servicios de maternidad de los centros hospitalarios de todo el mundo promover la lactancia y no aceptar el uso de fórmulas que sustituyan a la leche materna, así mismo, en el 2002 la Asamblea Mundial de la Salud y UNICEF respaldan la Estrategia Mundial para la Alimentación del Lactante y del Niño Pequeño, esta estrategia apoya la lactancia materna exclusiva por seis meses, seguida de alimentación complementaria oportuna mientras continua el amamantamiento por dos años y más(2).

A nivel mundial diferentes países han realizado investigaciones sobre la LME, un estudio realizado en Venezuela en el año 2011 demuestra que el $66,25 \%$ de las madres conocen la importancia de los beneficios de la lactancia materna tanto para la madre y para el(la) niño(a), y el $61,66 \%$ de las madres conocen sobre la técnica correcta para amamantar al(la)niño(a)(3); otro estudio realizado en Bolivia en el año 2013 revela que a pesar de que existe un alto porcentaje de madres (93\%) que aparentemente conocen algo de lactancia materna, tienen mala práctica (85\%)(4). Asimismo, en nuestro país se han realizado investigaciones; en Chiclayo en el año 2012 un estudio concluye que las madres tienen un nivel de conocimientos bajo referente a las prácticas adecuadas de lactancia materna(5).

Estos estudios previos, muestran las mejoras o deficiencias en conocimientos, actitudes y prácticas, los cuales son afectados por varios factores, así como el estudio de Zimmermann cols.(6); donde los conocimientos deficientes están en $12 \%$, Alvins y Briceño(3), encuentra que el $62 \%$ de las madres conocen sobre la técnica correcta para amamantar al niño, De Oliveira y Miranda(7) refieren de su población a pesar de haber recibido orientación sobre la lactancia Materna en su cuidado prenatal, un $71 \%$ no tenían conocimiento correcto con respecto a la frecuencia y duración de la lactancia materna, Flore(4) y González y Manzo(8), por su lado, si bien tienen buenos conocimientos no muestran al realizar una mala práctica. En estudios nacionales, Veramendi y cols.(9), describe que su población, tienen conocimientos de medio a bajo en $77 \%$, Vásquez(10) muestra que los conocimientos en lactancia materna, están 43\% nivel alto, 40\% nivel medio y $17 \%$ nivel bajo, en relación a sus actitudes, el 50\% muestra una actitud medianamente favorable, $30 \%$ favorable y $20 \%$ desfavorable, en cuanto a la práctica sobre lactancia materna, el $62 \%$ realiza practicas adecuadas y $38 \%$ practicas inadecuadas. Delgado, Mini(11) y Chavéz y cols.(12), muestran que la edad tiene relación con los conocimientos que tienen. Zimmermann(6), Mini y cols.(11), encuentra relación con la escolaridad, así como Cadena y Benavides(13) y Mini y cols.(11), su relación con la ocupación. Alwelare y cols.(14), menciona que el $51 \%$ cree que la fórmula infantil no es tan buena como la leche materna, por otra parte el $25 \%$ manifestó que la edad adecuada para incluir fórmula o cualquier otro alimento aparte de la leche materna es a los 5-6 meses de edad; un $43 \%$ opina que la lactancia materna complementada con leche de fórmula es la mejor opción para alimentar a sus bebés, y sólo un $22 \%$ está de acuerdo en que deben ser amamantados exclusivamente durante los primeros 6 meses de vida, en otros estudios como el de Flores(4), muestran que algunas etnias tienen la costumbre de dar mate de anís al recién nacido, agua, sopa de pescado, chila, somó y otros alimentos del lugar durante el tiempo de lactancia exclusiva. Otros autores, tales como
Vásquez(10) y Benjumea y cols.(15), muestran que un porcentaje considerable de las madres consideran que la lactancia materna proporciona beneficios tanto para la madre como para el bebé, incluso ayuda a combatir contra muchas enfermedades, mientras que otras madres, encontradas en el estudios de Gorrita y cols.(16), desconocen las ventajas y beneficios de la lactancia. Otro punto importante es que la madres no conocen el tiempo exclusivo de la lactancia materna y que prefieren dar leche de vaca en el tiempo exclusivo de la leche materna, así lo describe Ulenque y cols.(17), y que solo un $47 \%$ está de acuerdo con la lactancia materna exclusiva hasta los 6 meses de edad, Mini y cols.(11).

La lactancia materna es la forma natural de alimentación de la especie humana que proporciona el alimento ideal para el crecimiento y desarrollo del recién nacido, además ejerce una influencia biológica y afectiva inigualable, tanto en el niño como en la madre. Considerado un derecho humano fundamental que debe ser promovido y protegido por el Estado, la Sociedad, las Familias y la Comunidad.(18). La malnutrición ha sido relacionada con prácticas inadecuadas de alimentación, que ocurren durante el primer año de vida, tan sólo un $35 \%$ de los lactantes de todo el mundo son alimentados exclusivamente con leche materna durante los primeros cuatro meses de vida(19)

En el Perú, en 1996 y en el 2001 se restablecen algunos derechos de las madres trabajadoras, como son el descanso prenatal y postnatal y la hora diaria de lactancia hasta el año(20); Como todos sabemos, en la actualidad cada vez son más las mujeres que se integran al ámbito laboral, de esta manera se observa que cada vez, son más las mujeres que tienen que compartir su tiempo entre las actividades del hogar y las actividades del trabajo, inclusive durante su periodo de lactancia(21).

Es amplia la información acerca de lactancia materna; por lo que se debe instruir activamente a las madres y al personal de salud abarcando temas básicos que incluyan desde anatomía, fisiología, beneficios para la madre y el niño, entre otros.

La composición cambia según las necesidades del lactante, estos cambios se dan entre ciertos límites(22), está constituida por agua en su $88 \%$, proteínas, Nitrógeno no proteico, grasas, carbohidratos, vitaminas, minerales, factores inmunológicos(21).

La acción de amamantar proporciona calor, cercanía y contacto, lo que favorece el vínculo afectivo entre madre e hijo y puede ayudar al desarrollo físico y emocional del niño; también ayuda a la mandíbula del niño a desarrollar así como a los músculos tales como la lengua y los músculos de la trompa de Eustaquio, la leche materna protege contra muchas infecciones como otitis media, infecciones respiratorias, diarrea, caries dental, infección urinaria, sepsis neonatal y enterocolitis necrotizante(21,24), disminuye la posibilidad de leucemia y linfoma en la niñez, programa los sistemas corporales que pueden ayudar a la regulación y reducción del riesgo de obesidad y ciertas enfermedades cardiovasculares en la vida posterior, reduce el riesgo de alergias y de condiciones tales como diabetes juvenil, en familias con historia de estas condiciones. En la madre disminuye la mortalidad materna, el riesgo de cáncer de mama; Ofrece también beneficios sociales, no necesita preparación ni almacenamiento, es ecológica puesto que no tiene desperdicios ni requiere combustibles, que contaminen el medio ambiente(21).

Para el niño se observa mayor riesgo de mortalidad, puesto que los lactantes que no son amamantados durante los primeros 2 meses de vida tienen casi 6 veces más posibilidades de morir debido a enfermedades infecciosas, en comparación con los lactantes alimentados al pecho; los lactantes no amamantados entre los 2 y 3 meses tienen 4 veces más posibilidades de morir, en comparación con los alimentados al pecho; incluso los lactantes que no son amamantados a los 9 a 11 meses tiene $40 \%$ o más probabilidad de morir que los lactantes que reciben leche materna; los niños no amamantados presentan mayor riesgo de padecer enfermedades crónicas, como hipertensión arterial, colesterolemia y diabetes 
mellitus tipo 2, al no realizarse la práctica de la lactancia materna la madre presenta un mayor riesgo de padecer cáncer de seno y de ovario(2).

Así como los beneficios que nos otorgan la lactancia materna y los riesgos que existen en cuanto al no consumo de la misma; existen ciertas condiciones que impiden que los lactantes reciban tan preciado elemento para su adecuado crecimiento y desarrollo, donde se ha demostrado la transmisión del VIH tipo 1 por la leche materna. Los niños afectos de Galactosemia deberán recibir una dieta sin lactosa ni galactosa por la que habrá que excluir la leche y todos los productos lácteos y sustituirlos por fórmulas especiales. El consumo de drogas como heroína, cocaína, anfetamina y marihuana también es incompatible con la lactancia materna. En la infección materna por Citomegalovirus, casi todas las mujeres seropositivas sufren reactivación y excretan el virus durante la lactancia, de la misma manera madres portadoras del virus de la Hepatitis B (HVB), las usuarias de medicamentos radiactivos (quimioterapia).

Por todo lo expuesto, nos planteamos la siguiente interrogante ¿Cuál es el nivel de conocimientos, actitudes y prácticas de lactancia materna de las madres que acuden al control prenatal del Hospital Regional de Ica en Enero del 2017? Asimismo, este estudio tiene como objetivo evaluar los conocimientos, identificar las actitudes, determinar las prácticas y revisar la congruencia entre estos tres puntos. Respecto a la hipótesis cabe resaltar que por ser de características netamente de tipo descriptiva este estudio no presenta hipótesis.

\section{MATERIAL Y MÉTODOS}

Se realizó una Investigación con nivel observacional, tipo prospectivo, transversal y descriptivo. En una población constituida por madres que acuden al control prenatal del Hospital Regional de Ica durante el mes de Enero del año 2017.

Previo a la obtención de la muestra se obtuvo datos estadísticos del Hospital que nos dieron a conocer que el promedio de pacientes que se atienen al mes en dicho consultorio es de 360 , posterior a ello se procedió a encuestar de 160 pacientes que cumplían con nuestros criterios de inclusión y exclusión durante el mes del estudio, posterior a ello se realizó el cálculo muestral con las siguiente formula. $n=\frac{Z_{\alpha}^{2} \times p \times q \times N}{Z_{\alpha}^{2} \times p \times q \times N \times e^{2}:}$ Donde: $\mathrm{n}$ : tamaño de muestra z: valor correspondiente a la distribución de gauss, $z \alpha=0.05=1.96$ p: probabilidad de ocurrencia $=0.5$ q: probabilidad de no ocurrencia $=1-\mathrm{p}=0.5 \mathrm{~N}$ : población $=360$ e: error que se prevé cometer si es del $5 \%$, e $=0.05 \quad n=\frac{1.96^{2} \times 0.5 \times 0.5 \times 136}{1.96^{2} \times 0.5 \times 0.5+360 \times 0.05^{2}} \quad n=100.44, \quad$ por lo que se tomó en cuenta como muestra del estudio a 100 pacientes; una vez obtenido la muestra se procedió a escoger al azar las encuesta mediante programa Excel 2013 cumpliendo de esta manera con el muestreo probabilístico.

Se realizó es estudio en madres que estuvieron esperando su turno en el consultorio de control prenatal del Hospital Regional de Ica, que estuviesen dispuestas a aceptar participar del estudio firmando el consentimiento informado, las madres que estén dispuestas a colaborar con las encuestas, madres que hablen castellano y que al menos tengan un hijo; se excluyeron del estudio las madres que no respondan en forma completa a la encuesta, las que no deseen participar, las primigestas y las que no quisieron firmar el consentimiento informado.

La técnica de recolección que se utilizó es la encuesta, y en el procesamiento de datos y análisis estadísticos se utilizó el programa estadístico SPSS 24.

Para la recolección de los datos se usó una encuesta la cual estuvo validada por un estudio previo realizado en el hospital Regional Docente las Mercedes-Chiclayo en el año 2012 en el cual evalúan nivel de conocimientos de las madres adolescentes en la práctica de la lactancia materna exclusiva; el cual contiene dieciocho ítems, de las cuales dos ítems corresponden a datos sociodemográficos de la encuestada, nueve ítems en forma de preguntas están dirigidas a evaluar conocimientos, tres ítems en forma de preguntas dirigidas a evaluar actitudes y cuatro ítems destinados a evaluar prácticas, dos de los cuales fueron evaluados mediante el uso de una maqueta.

Una vez que se obtuvo toda la información de las diferentes variables, se procedió a transferir la información a una base de datos elaborada mediante el programa SPSS 24 , con este programa se procedió a realizar:

Análisis univariado, se realizó análisis de tendencia central, se calculó las frecuencias y porcentajes para las variables cualitativas. Para las variables cuantitativas se calculó las medias, medianas y moda.

Análisis bivariado, se aplicó análisis correlacional para detectar grado de asociación, mediante tablas de contingencia, teniendo presente las variables dependientes, para la medida de asociación se utilizó la prueba de chi cuadrado con un nivel de confianza del $95 \%$.

En el estudio realizado las variables dependientes fueron categorizadas de la siguiente manera:

1. Conocimientos: se evaluó la variables mediante 8 preguntas, clasificándose como correcto las que contestaron $\geq 5$ preguntas e incorrecto $<5$ preguntas.

2. Actitudes: se evaluó la variable mediante 2 preguntas, clasificándose como adecuada las que contestaron 2 preguntas e inadecuada $\leq 1$ pregunta.

3. Practicas: se evaluó la variable mediante 2 ítems, clasificándose como buenas 2 puntos y malas $\leq 1$ punto.

En el presente estudio se contó con la aprobación del comité de ética de investigación del Hospital Regional de Ica, asimismo se respetaran los principios de confidencialidad y la información obtenida será utilizada estricta y exclusivamente para los fines del estudio en concordancia con la declaración de Helsinki y el informe de Belmont. Finalmente los resultados se presentaran sin incurrir en falsificación ni plagio y declarando que no hay ningún conflicto de interés.

\section{RESULTADOS}

En el estudio realizado se aprecia que la distribución de la edad de las mujeres que fueron encuestadas seguía una distribución que aparentaba ser normal. Al realizar las mediciones de tendencia central se obtuvo mediana, media y moda de 26 , por lo cual se concluye que la distribución de edades sigue una distribución normal, por lo que los resultados que deriven de ello serían consistentes como muestra el grafico $\mathrm{N}^{\circ} 1$.

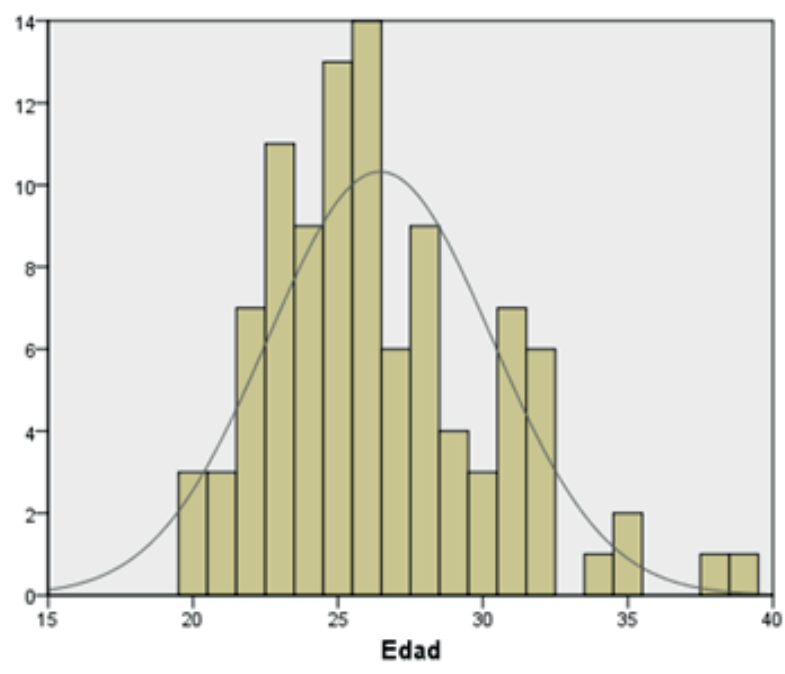

Gráfico N 1. Distribución de edad DS: 3.87 , Me: 26.45 
Siendo La mediana de edad es 26 y la mayoría de edades oscilan entre 20 y 35, concentrándose con mayor porcentaje entre 25 y 26. Los extremos de edades de las mujeres encuestadas son 20 años para el extremo inferior y 39 años como el extremo superior de edad.

Respecto al nivel de educación de las mujeres encuestadas,18\% tenía nivel universitario, $20 \%$ nivel primario y $62 \%$ nivel secundario; respecto a su ocupación el $53 \%$ era ama de casa, el 15\% estudiante, el $29 \%$ era ama de casa y trabajaba a la vez y el $3 \%$ estudiaba y trabajaba a la vez; respecto a la edad de su último hijo, lo cual indirectamente nos mostraba cuánto tiempo había transcurrido desde su última experiencia de dar lactancia la mediana de edad del último hijo fue de 3 años, que el $75 \%$ de todas las mujeres encuestadas tuvo su último hijo hace 4.75 años y que las edades extremas del último hijo fueron 1 año para el extremo inferior y 11 años para el extremo superior.

Con respecto a los conocimientos sobre que es la lactancia materna exclusiva vemos que un $83 \%$ de mujeres encuestadas dice que es amamantamiento exclusivo con leche materna, $12 \%$ manifiesta que es agregar otras sustancias a la lactancia materna y en $5 \%$ se desconoce que sobre el tema tal y como muestra la Tabla $\mathrm{N}^{\circ} 1$.

Tabla 1.

\section{Lactancia Materna Exclusiva}

\begin{tabular}{lcc}
\hline & Frecuencia & Porcentaje \\
\hline $\begin{array}{lcc}\text { Amamantamiento exclusivo con la } \\
\text { leche materna }\end{array}$ & 83 & 83,0 \\
$\begin{array}{l}\text { Amamantamiento con la leche } \\
\text { materna y otras sustancias }\end{array}$ & 12 & 12,0 \\
Desconoce & 5 & 5,0 \\
Total & 100 & 100,0
\end{tabular}

Sobre la importancia de la lactancia materna exclusiva el $70 \%$ manifestó que la leche materna contiene todos los nutrientes necesarios hasta los 6 meses de edad, el 17\% que mantiene el sistema inmunológico, el $9 \%$ que es la única leche que tolera el bebé y el $4 \%$ desconoce la razón; sobre los beneficios de la lactancia materna exclusiva el $75 \%$ opina que la leche materna garantiza escenarios beneficiosos para el bebé (garantiza el crecimiento, desarrollo e inteligencia del niño, disminuye el riesgo de contraer enfermedades, fortalece el vínculo afectivo y disminuye la hemorragia posparto, la anemia y la mortalidad materna), el $25 \%$ opina que sólo ayuda a que el bebé no llore mucho y para que la madre esté tranquila, sobre si la lactancia materna exclusiva tiene ventajas o no el $33 \%$ opina que la lactancia materna exclusiva tiene ventajas, el $42 \%$ opina que no tiene ventajas y el $25 \%$ desconoce si la lactancia materna exclusiva tiene ventajas; en tanto a cuándo deben empezar a dar lactancia materna a sus hijos el $65 \%$ manifiestan que deberían iniciarse la lactancia materna inmediatamente al nacer su hijo, 35\% a las 24 horas del nacimiento del hijo; respecto a la frecuencia que deben brindarse la lactancia materna el 1\% opinó cuando tenía leche o calostro, el $44 \%$ cuando el bebé lloraba, el $2 \%$ cada 3 horas, el $53 \%$ cada vez que el hijo quería, sobre el motivo o razón por la cual deben brindar lactancia materna a sus hijos, en términos de alimentación el $26 \%$ opinaron que no puede tomar otro alimento y el $74 \%$ opinaron que la leche materna los nutre mejor y protege de enfermedades; respecto al mínimo de tiempo que se debe brindar la lactancia materna exclusiva. El 10\% opinaron hasta los 4 meses, el $3 \%$ hasta los 5 meses, el $82 \%$ hasta los 6 meses y el $5 \%$ desconocían el tiempo mínimo para dar lactancia materna exclusiva.
Respecto a la actitud de las mujeres encuestadas frente al tipo de leche que administró a sus hijos el $68 \%$ sólo administró leche materna, el $4 \%$ sólo otro tipo de leche, el $28 \%$ administró una combinación de leche materna más otro tipo de leche, al conocer si en algún momento, dentro de los 06 primeros meses de vida, dejaron de brindar de la lactancia materna, un $68 \%$ de las madres menciono no haberla suspendido en ningún momento, de las 32 mujeres que dejaron de dar la lactancia materna durante los 6 primeros meses, el $34 \%$ fue por continuar sus estudios y el $15 \%$ por falta de leche. Quizás esto lleve a analizar que quizás la mayoría de mujeres que dejó de dar lactancia materna pertenecían al grupo que estudiaba o estudiaba y trabajaba.

En cuanto a las prácticas se obtuvo que sólo el $26 \%$ realizaban la práctica del posicionamiento del bebé, el $44 \%$ practicaban la postura adecuada de la madre y el $31 \%$ practicaba la coherencia y coordinación entre lo que manifestaba y realizaba.

Se obtuvo también la opinión de las mujeres encuestadas sobre que orientación habían recibido del establecimiento de salud de las cuales 53 mujeres refirieron haber recibido orientación sobre la posición del cuerpo para amamantar, 69 sobre la duración de la lactancia materna exclusiva, 50 sobre dar de lactar cada vez que el bebé quiera y un 40 sobre la colocación y ubicación cuerpo a cuerpo del bebé al seno de la madre.

En resumen según las respuestas de las mujeres encuestadas, el grado de conocimientos, actitudes y prácticas sobre lactancia materna. A pesar de que el $65 \%$ de las mujeres encuestadas tenían buenos conocimientos sobre lactancia materna, sólo el 13\% lograron adecuadas actitudes frente a la misma y el 39\% realizaban buenas prácticas sobre la lactancia materna tal como nos muestra el grafico $\mathrm{N}^{\circ} 2$.

\section{Gráfico N2. Lactancia Materna}

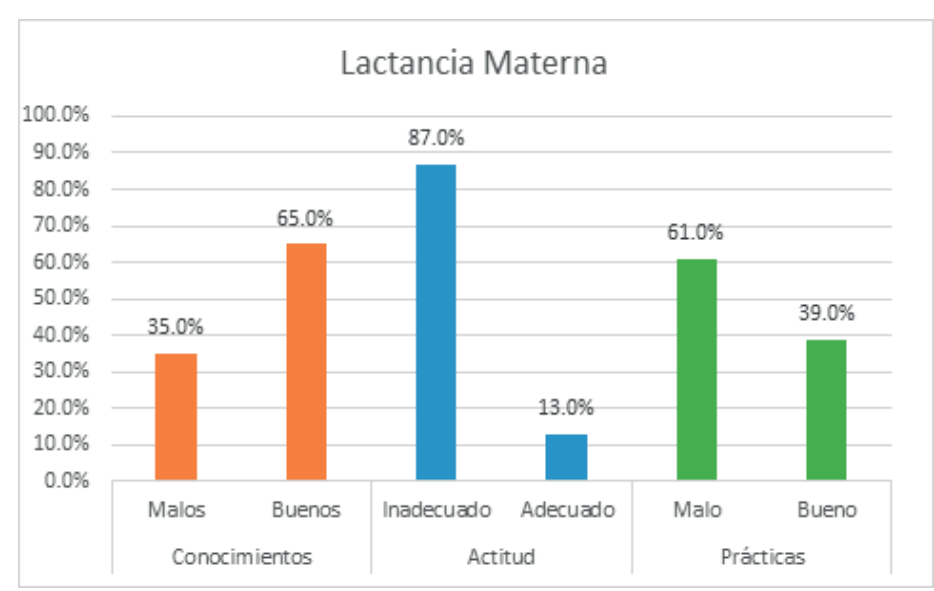

Conocimientos malos: $<5$ preguntas respondidas correctamente, buenos: $\geq 5$ preguntas respondidas correctamente. Actitudes inadecuadas: $\leq 1$ preguntas respondidas incorrectamente, adecuadas: 2 preguntas respondidas correctamente. Prácticas inadecuadas: $\leq 1$ preguntas respondidas incorrectamente, adecuadas: 2 preguntas respondidas correctamente.

Al realizar el cruce de variables dependientes e independientes vemos que el análisis de asociación entre edad de las mujeres encuestadas y los conocimientos sobre lactancia materna aplicando chi cuadrado con IC $95 \%$ se obtiene un chi cuadrado 0.183 lo cual nos dice que no hay relación entre variables en ningún sentido y que tampoco alcanza un nivel significativo $(p=0,67)$.

La relación entre las variables de nivel de escolaridad con el nivel de conocimientos resulta un chi cuadrado de 10,496 lo cual nos dice que si existe una relación lineal entre nivel de escolaridad con el nivel de conocimientos (a mayor nivel de escolaridad, mayor nivel 
Tabla 2.

Conocimientos en lactancia materna - Nivel de escolaridad

\begin{tabular}{|c|c|c|c|c|c|c|}
\hline & & & \multicolumn{2}{|c|}{ Conocimientos } & \multirow{2}{*}{ Total } & \multirow{2}{*}{$\mathrm{p}$ - valor } \\
\hline & & & Incorrectos & Correctos & & \\
\hline \multirow{6}{*}{$\begin{array}{l}\text { Nivel de } \\
\text { Escolaridad }\end{array}$} & \multirow{2}{*}{ Primaria } & $\mathrm{N}$ & 11 & 9 & 20 & \\
\hline & & $\%$ & $55,0 \%$ & $45,0 \%$ & $100,0 \%$ & \\
\hline & \multirow{2}{*}{ Secundaria } & N & 23 & 39 & 62 & \\
\hline & & $\%$ & $37,1 \%$ & $62,9 \%$ & $100,0 \%$ & 0.005 \\
\hline & \multirow{2}{*}{ Universidad } & $\mathrm{N}$ & 1 & 17 & 18 & \\
\hline & & $\%$ & $5,6 \%$ & $94,4 \%$ & $100,0 \%$ & \\
\hline \multirow{2}{*}{ Total } & & N & 35 & 65 & 100 & \\
\hline & & $\%$ & $35,0 \%$ & $65,0 \%$ & $100,0 \%$ & \\
\hline
\end{tabular}

Se aprecia la relación entre las variables de ocupación y conocimientos (chi cuadrado de 9,104) es decir a mayor ocupación mayor nivel de conocimientos, se alcanza un nivel estadístico significativo $(p=0,028)$ como se muestra en la tabla $\mathrm{N}^{\circ} 3$.

Tabla 3.

Conocimientos en lactancia materna - Ocupación

\begin{tabular}{|c|c|c|c|c|c|c|}
\hline & & & \multicolumn{2}{|c|}{ Conocimientos } & \multirow{2}{*}{ Total } & \multirow{2}{*}{ p- valor } \\
\hline & & & Incorrectos & Correctos & & \\
\hline \multirow{6}{*}{ Ocupación } & \multirow{2}{*}{ Ama de casa } & $\mathrm{N}$ & 25 & 28 & 53 & \multirow{10}{*}{0.028} \\
\hline & & $\%$ & $47,2 \%$ & $52,8 \%$ & $100,0 \%$ & \\
\hline & \multirow{2}{*}{ Estudiante } & $\mathrm{N}$ & 5 & 10 & 15 & \\
\hline & & $\%$ & $33,3 \%$ & $66,7 \%$ & $100,0 \%$ & \\
\hline & \multirow{2}{*}{$\begin{array}{l}\text { Ama de casa - } \\
\text { trabaja }\end{array}$} & $\mathrm{N}$ & 5 & 24 & 29 & \\
\hline & & $\%$ & $17,2 \%$ & $82,8 \%$ & $100,0 \%$ & \\
\hline \multirow{4}{*}{ Total } & \multirow{4}{*}{ Estudia - trabaja } & $\mathrm{N}$ & 0 & 3 & 3 & \\
\hline & & $\%$ & $0,0 \%$ & $100,0 \%$ & $100,0 \%$ & \\
\hline & & $\mathrm{N}$ & 35 & 65 & 100 & \\
\hline & & $\%$ & $35,0 \%$ & $65,0 \%$ & $100,0 \%$ & \\
\hline
\end{tabular}

Con respecto a la relación entre variables de edad y actitudes frente la lactancia materna. Se obtiene un chi cuadrado de 0,531 lo cual nos dice que no hay relación entre variables y al obtener $p=0,466$ demuestra que tampoco hay significancia estadística. Vemos también que se aprecia la relación entre variables de nivel de escolaridad y actitudes frente a la lactancia materna obteniéndose un chi cuadrado de 1,686 lo que significa que hay asociación entre variables (aunque débil). Es importante remarcar que la distribución de mujeres en cada grupo de nivel de escolaridad no es homogénea. Al obtener una $p=0,430$ se concluye que está asociación de variables no alcanza un nivel estadístico significativo como nos muestra la tabla $\mathrm{N}^{\circ} 4$.

Tabla 4.

\section{Actitudes en lactancia materna - Nivel de escolaridad}

\begin{tabular}{|c|c|c|c|c|c|c|}
\hline & & & \multicolumn{2}{|c|}{ Actitudes } & \multirow{2}{*}{ Total } & \multirow{2}{*}{ p- valor } \\
\hline & & & Incorrectos & Correctos & & \\
\hline \multirow{6}{*}{ Ocupación } & \multirow{2}{*}{ Primaria } & $\mathrm{N}$ & 16 & 4 & 20 & \\
\hline & & $\%$ & $18,4 \%$ & $30,8 \%$ & $20,0 \%$ & \\
\hline & \multirow{2}{*}{ Secundaria } & $\mathrm{N}$ & 56 & 6 & 62 & \\
\hline & & $\%$ & $64,4 \%$ & $46,2 \%$ & $62,0 \%$ & 0.43 \\
\hline & \multirow{2}{*}{ Universidad } & $\mathrm{N}$ & 15 & 3 & 18 & \\
\hline & & $\%$ & $17,2 \%$ & $23,1 \%$ & $18,0 \%$ & \\
\hline \multirow{2}{*}{ Total } & & $\mathrm{N}$ & 87 & 13 & 100 & \\
\hline & & $\%$ & $100,0 \%$ & $100,0 \%$ & $100,0 \%$ & \\
\hline
\end{tabular}


Respecto a la ocupación de la madre con actitudes frente a la lactancia materna se obtiene el chi cuadrado de 1,647 lo que demuestra asociación débil (a mayor ocupación, mejores actitudes), al obtener una $\mathrm{p}=0,65$, la relación no alcanza significancia estadística.

Con respecto a la asociación entre variables de edad de la madre y las Prácticas sobre lactancia materna se obtiene un chi cuadrado de 2.270 que demuestra una asociación débil entre variables (a más jóvenes, mejores actitudes) pero al obtener una $\mathrm{p}=0,132$ no alcanza significancia estadística.

Vemos que la relación entre variables de nivel de escolaridad y prácticas en lactancia materna se obtuvo un chi cuadrado de 8,262 lo cual nos dice que si existe relación entre variables (a menor grado de instrucción o escolaridad mejores prácticas sobre lactancia materna) y una $p=0,016$ que nos demuestra que si hay significancia estadística como nos muestra la tabla $\mathrm{N}^{\circ} 5$.

Tabla 5.

Prácticas en lactancia materna - Nivel de escolaridad

\begin{tabular}{|c|c|c|c|c|c|c|}
\hline & & & \multicolumn{2}{|c|}{ Actitudes } & \multirow{2}{*}{ Total } & \multirow{2}{*}{ p- valor } \\
\hline & & & Incorrectos & Correctos & & \\
\hline \multirow{6}{*}{ Ocupación } & \multirow{2}{*}{ Primaria } & $\mathrm{N}$ & 9 & 11 & 20 & \\
\hline & & $\%$ & $45,0 \%$ & $55,0 \%$ & $100,0 \%$ & \\
\hline & \multirow{2}{*}{ Secundaria } & $\mathrm{N}$ & 36 & 26 & 62 & \\
\hline & & $\%$ & $58,1 \%$ & $41,9 \%$ & $100,0 \%$ & 0.016 \\
\hline & \multirow{2}{*}{ Universidad } & $\mathrm{N}$ & 16 & 2 & 18 & \\
\hline & & $\%$ & $88,9 \%$ & $11,1 \%$ & $100,0 \%$ & \\
\hline \multirow{2}{*}{ Total } & & $\mathrm{N}$ & 61 & 39 & 100 & \\
\hline & & $\%$ & $61,0 \%$ & $39,0 \%$ & $100,0 \%$ & \\
\hline
\end{tabular}

Con respecto la asociación entre variables de ocupación de la madre y prácticas sobre lactancia materna se obtuvo un chi cuadrado de 3,064 lo que demuestra una relación débil entre las variables (a menor ocupación, mejores prácticas sobre lactancia materna) pero la $\mathrm{p}=0.38$ nos dice que dicha asociación no alcanza valores estadísticos significativos.

\section{DISCUSIÓN}

Entonces en nuestro estudio encontramos que el $65 \%$ de las madres presenta conocimientos correctos sobre LME. Resultados similares se aprecian en otros estudios, tales como Zimmermann cols 6 en el que el $88 \%$ presento conocimientos adecuados, y Flores 4 en su estudio, encontró que un $93 \%$ de las madres tenían conocimientos buenos. Resultados opuestos se obtuvieron en el trabajo realizado por Gonzalez y Manso8 donde el 59\% presentaron conocimientos incorrectos, además Guerrero y Vera25, en su trabajo de investigación, obtienen que un $55 \%$ tiene un nivel malo de conocimiento. En estudios nacionales, Veramendi y cols.9, describe que su población, tienen conocimientos de medio a bajo en $77 \%$, mientras Vásquez10 en su estudio realizado en Lima, describe que solo el $17 \%$ de las madres tienen un nivel bajo de conocimientos de LME.

La mediana en edad es de 26 años, con una mínima de 20 años y máxima de 39 años, sobre el grupo que tienen menos de 27 años, aquellas que tienen conocimientos incorrectos representan un $63 \%$, Delgado5 en su trabajo realizado en Chiclayo, un $48 \%$ de madres adolescentes presentan un nivel medio de conocimientos y solo un $11 \%$ nivel alto.

Sobre la importancia de la lactancia materna exclusiva un 70\% reconoce que sólo la leche materna contiene todos los nutrientes necesarios para el niño hasta los 06 meses de edad, dichos resultados coinciden con los obtenidos en la investigación de Cadena y Benavides 13 donde un $75 \%$ de las madres están concientizadas sobre la importancia de la LME.

En cuanto a los beneficios de la lactancia materna exclusiva tanto para el niño como para la madre, la mayoría de las madres (75\%) posee conocimiento sobre estos, lo cual concuerda con los resultados obtenidos en el estudio realizado por Alvins y Briceño3 $(66,25 \%)$.

Al preguntar sobre el motivo de la ingesta de la leche materna, un $74 \%$ de las madres refieren que la leche materna nutre mejor que otros alimentos y protege contra enfermedades, resultados similares presentaron Alwelare, Al-Harthy14 en su trabajo en el cual la mayoría de las mujeres $(51,0 \%)$ cree que la fórmula infantil no es tan buena como la leche materna, sin embargo el $42,7 \%$ opina que la lactancia materna complementada con leche de fórmula es la mejor opción para alimentar a sus bebés.

El $82 \%$ de las madres sabe que como mínimo debe brindar la lactancia materna exclusiva hasta los 06 meses de vida, resultados similares hallamos en el trabajo de Cadena y Benavides13 (91\%), y en el trabajo de De Oliveira y Miranda7 (72,91\%); pero se obtuvieron datos opuestos en el trabajo de Flores4, donde el $85 \%$ desconocía el tiempo adecuado de LME; además en el trabajo de Alwelare, Al-Harthy14 sólo el 22,0\% de las mujeres está de acuerdo en que los bebés deben ser amamantados exclusivamente durante los primeros 6 meses de vida.

En nuestro trabajo se observa que el mayor número de madres con conocimientos incorrectos tienen un nivel de escolaridad primario, y el mayor número de madres con conocimientos correctos tienen un nivel de escolaridad universitario, estos resultados concuerdan con los obtenidos por Zimmermann y Col6 quienes obtuvieron que el mayor nivel de escolarización se asocia con conocimientos más extensos y seguros acerca de LME.

Respecto a la actitud que tienen las madres de realizar la LME solo el $13 \%$ de las madres tienen adecuadas actitudes, al conocer si en algún momento, dentro de los 06 primeros meses de vida, dejaron de brindar de la lactancia materna, un $32 \%$ de las madres menciono haberla suspendido en algún momento y al indagar las causas de la separación de la lactancia materna, el $47 \%$ mencionaron que fue por falta de leche, dichas causas concuerdan con los obtenidos por Cadena y Benavides 13 donde el $82 \%$ de las madres abandonaron la lactancia materna por falta de leche.

Al examinar sobre el aspecto práctico de la lactancia materna, el $26 \%$ presenta un posicionamiento adecuado del bebé y el $44 \%$ de las madres mostró una postura adecuada, en su trabajo Vásquez10 encontró que Un porcentaje considerable de madres tiene practicas adecuadas en lactancia materna ya que se acomoda adecuadamente en la silla para dar de lactar a su bebe así como posiciona adecuadamente al bebé.

\section{CONLUSIONES}

En general vemos que las gestantes tienen buenos conocimientos en lactancia materna exclusiva, pero cuando estos conocimientos se deben traducir en actitudes correctas sobre lactancia materna y buenas prácticas de amamantamiento, no se obtienen buenos resultados. Por lo cual concluimos que las gestantes a pesar de 
tener buenos conocimientos sobre lactancia materna no los pueden aplicar en la práctica. Se identificó al nivel de escolaridad como factor importante para los conocimientos y actitudes sobre la lactancia materna en las mujeres.

En algunos análisis bivariados en los cuales se encuentra asociación de variables pero sin significación estadística (nivel de escolaridad y actitudes, ocupación y actitudes, edad de madre y prácticas, ocupación y prácticas) se debe realizar otros estudios con mayor marco muestras o distribución homogénea de grupos. También realizar análisis multivariados para ver la asociación neta de cada variable sobre la otra y mejorar las frecuencias, ya que si éstas son muy bajas (menores de 5 ) se debe cambiar las pruebas estadísticas.

\section{BIBLIOGRAFÍA}

1. Aguilar P.; Ayerbe L. Lactancia materna basada en la evidencia. Revista de Enfermería Garnata(Granada). 2012; 91(22): 41-45

2. Fondo de las Naciones Unidas para la Infancia, Ministerio de Salud, Caja Costarricense del Seguro Social, Instituto Nacional de Estadística y Censos. Análisis del Módulo de Lactancia Materna: Encuesta Nacional de Hogares, informe final 2010. San José; 2011.

3. Alvins C., Briceño A. Conocimientos de las Madres Adolescentes sobre los beneficios de la Lactancia Materna Exclusiva, Chile, 2011. [Internet] 2011 [acceso 20 de Junio de 2014]. Disponible en: http://bibmed.ucla.edu.ve/DB/bmucla/edocs/textocompleto/TIEWS125A582011.pdf

4. Flores Pacheco M. Conocimiento y práctica en lactancia materna exclusiva en las etnias del municipio de Rurrenabaque 2011 [tesis doctoral]. La Paz: Universidad Mayor de San Andrés; 2014.

5. Delgado L., Nombera F. Nivel de conocimientos de las madres adolescentes en la práctica de la lactancia materna exclusiva HRDLM - Chiclayo, 2012 [tesis doctoral]. Chiclayo: Servicio de Publicaciones e Intercambio Científico, Universidad Católica Santo Toribio de Mogrovejo; 2013.

6. Zimmermann C., Medina Pinto S., Ortiz Gavilán A., Miño C. Conocimiento sobre lactancia materna en puérperas de la ciudad de Resistencia en el año 2010. Revista de Posgrado de la VI Cátedra de Medicina (Argentina). 2011; 207: 1-5.

7. De Oliveira Fonseca M., Miranda Parreira B. Aleitamento materno: Conhecimento de Maes admitidas no alojamento conjunto de um Hospital Universitário. Cienc Cuid Saude (Brasil). 2011 Jan/Mar; 10(1):141-149

8. Gonzáles Reyes M., Manzo Villón C. Conocimientos sobre la importancia de la lactancia materna que tienen las embarazadas primigestas del Hospital "Dr. Liborio Panchana Sotomayor" 2011 2012 [tesis doctoral]. La Libertad: Universidad Estatal Península de Santa Elena; 2012.

9. Veramendi Espinoza LE, Zafra-Tanaka JH, Ugaz-Soto LM, Villa Santiago NM, Torres-Bravo LA, Pinto-Arica AA, Moras Rosado MM. CIMEL. Conocimientos, actitudes y prácticas de lactancia materna en madres de niños de Comunidad rural peruana y su asociación con la diarrea aguda infecciosa.(Lima). 2012; 17(2): 82-88

10. Vásquez Delgado R. Conocimiento, actitudes y prácticas en lactancia materna de las madres que acuden al Centro de Salud San Luis, 2009 [tesis doctoral].Lima: Servicio de Publicaciones e Intercambio Científico, Universidad Nacional Mayor de San Marcos; 2012.

11. MINI, Elsy et al. Conocimientos, actitudes y prácticas sobre la lactancia materna exclusiva, en madres lactantes con niños meno res de un año, INMP-2011. Anales de la Facultad de Medicina, 2013; vol 73: pag S69, ISSN 1609-9419. Disponible en: <http://revistasinvestigacion.unmsm.edu.pe/index.php/anales/article/view/2269>.

12. Chavéz Loyola A., Dávila Escudero R., Pesántez Bermeo A. Conocimientos, actitudes y prácticas sobre lactancia materna en madres lactantes. Hospitales Cantonales de Paute, Santa Isabel y Centro de Salud de Nabón, 2013 [tesis doctoral].Cuenca: Universidad de Cuenca; 2014.

13. Cadena L., Benavides D.; Conocimientos, aptitudes y prácticas sobre lactancia materna en madres con niños de cero a 1 año de edad que acuden al centro de salud no. 1 de la Ciudad de Tulcán Provincia del Carchi de Enero - Agosto del 2010 [tesis doctoral]. Ibarra: Universidad Técnica del Norte; 2010.

14. Alwelare, Al-Harthy. Breastfeeding knowledge and attitude among Saudi women in Central Saudi Arabia. Rev Saudi Med J 2010; 31(2): 193-198.

15. Benjumea MV, Falla NC, Jurado L. Conocimientos y prácticas de gestantes y cuidadores sobre lactancia materna en cinco municipios caldenses. Rev Hacia promoc salud (Colombia). 2013; 18(2):66-78

16. Gorrita Pérez R., Brito Linares D., Ravelo Rodríguez Y., Ruiz Hernández E. Conocimientos de futuras madres sobre la lactancia materna y sus ventajas. Revista de ciencias médicas (La Habana). 2014; 20(1): 77-88.

17. Ulunque Unzueta A., Uria Guisbert E., Vairo Gamarra J., Vargas Almendras A., Santander López A. Conocimiento sobre Lactancia Materna en Madres de los Centros de Salud Pacata y Jaihuayco del 2008 al 2009. Rev Cient Cienc Med. (Bolivia). 2010; 13(2): 73-6.

18. Ministerio de la mujer y poblaciones vulnerables. Lactancia materna: un derecho universal. Lima: Ministerio de la mujer y poblaciones vulnerables; 2013.

19. Organización Panamericana de la Salud. Semana Mundial de la Lactancia Materna, 2012 Comprendiendo el Pasado - Planificando el Futuro Celebración del $10^{\circ}$ aniversario de la Estrategia Mundial para la Alimentación del Lactante y del Niño Pequeño de la OMS/UNICEF. 20. Ministerio de Salud. Documento técnico curso taller "Promoción y apoyo a la lactancia materna en establecimientos de salud amigos de la madre y el niño". Lima: Depósito Legal en la Biblioteca Nacional del Perú № - 2009-11279; 2009.

21. Alianza Mundial pro Lactancia Materna. Semana Mundial de Lactancia Materna 2015. . [monografía en Internet]. Penang; 2015[acceso 21 de Septiembre del 2015]. Disponible en : http://worldbreastfeedingweek.org/pdf/wbw2015-af-spa.pdf

22. Instituto Nacional de Estadística e Informática. Indicadores de Resultados de los Programas Estratégicos, 2011 [sede Web]. Lima; 2010- [acceso 14 de Marzo del 2014]. Disponible en : http://www.inei.gob.pe

23. Comité de lactancia materna de la Asociación Española de Pediatría. Lactancia materna a demanda. [monografía en Internet]. Madrid: Asociación Española de Pediatría; 2013 [acceso 15 de Junio del 2014]. Disponible en: http://www.aeped.es/sites/default/files/d o c u m e n t o s / l a c t a n c i a _ materna_a_demanda_recomendaciones_clm.pdf

24. Moreno J., Galiano M., Dalmau J. ¿Por qué dudamos de si la leche de vaca es buena para los niños? Acta Pediatr Esp (Madrid). 2012; 70(9): 369-375 